\title{
Traitement insecticide des bovins contre les glossines par pédiluve en conditions expérimentales
}

\author{
J. Bouyer ${ }^{1,2 *}$ F. Stachurski ${ }^{1,3}$ A. Gouro ${ }^{2}$ R. Lancelot ${ }^{1}$
}

\begin{abstract}
Mots-clés
Bovin - Glossina - Pyréthrine de synthèse - Contrôle de maladie Pulvérisation - Trypanosomose Dispositif expérimental.
\end{abstract}

\begin{abstract}
Résumé
L'étude du comportement des arthropodes vecteurs peut aboutir à la mise au point de méthodes de lutte innovantes et économiques pour les éleveurs. Ainsi, le traitement ciblé de l'extrémité des membres des bovins par pédiluve acaricide permet-il d'éliminer les tiques Amblyomma variegatum (Acari : Ixodidae) capturées au pâturage avant qu'elles n'accèdent à leurs sites de fixation définitifs. Les auteurs ont récemment montré qu'avec une solution d' $\alpha$-cyperméthrine, le traitement pédiluve était aussi efficace qu'une pulvérisation intégrale pour lutter contre Glossina tachinoides Westwood, 1850 (Diptera, Glossinidae) et G. palpalis gambiensis Vanderplank 1949. Ils ont comparé ici en conditions expérimentales (cages et étable moustiquaire) l'efficacité d'une formulation de deltaméthrine (Vectocid) administrée par pulvérisation intégrale, partielle, ou par pédiluve, efficacité qui s'est traduite par la paralysie de ces deux espèces et de G. morsitans submorsitans Newstead 1910 lorsqu'elles ont été mises en présence des animaux traités. Lors de l'exposition aux glossines en cage, le taux de paralysie des glossines exposées aux bovins imprégnés par pédiluve a été similaire à celui obtenu par pulvérisation partielle $(p=0,062)$ mais inférieur à celui obtenu par aspersion complète $\left(p<10^{-3}\right)$. En volières, il a été inférieur à celui des deux autres modalités de traitement $\left(p<10^{-3}\right)$. Malgré cela, la mortalité due au pédiluve, calculée sur 15 jours avec une fréquence de traitement de trois jours, est restée supérieure à celle nécessaire pour obtenir l'extinction d'une population isolée de glossines ( 3 p. $100 \mathrm{j}^{-1}$ ). Cette technique pourrait donc être efficace dans la lutte préventive contre les trypanosomoses bovines transmises par les glossines dans certaines conditions.
\end{abstract}

\section{INTRODUCTION}

L'étude du comportement des arthropodes vecteurs de maladies infectieuses ou parasitaires peut aboutir à la mise au point de méthodes de lutte innovantes et économiques pour les éleveurs. L'application d'une suspension aqueuse de pyréthrinoïdes sur la partie distale des membres des bovins à l'aide d'un pédiluve permet ainsi de tuer les tiques de l'espèce Amblyomma variegatum (Acari : Ixodidae) avant qu'elles ne se fixent définitivement sur

\footnotetext{
1. Cirad, UMR Contrôle des maladies animales exotiques et émergentes, TAA-15/B, Campus international de Baillarguet, Montpellier Cedex 5, F-34398 France. 2. Cirdes, UR Elevage et environnement, Bobo Dioulasso, Burkina Faso. 3. Fofifa, département de Recherches zootechniques et vétérinaires, Antananarivo, Madagascar

* Auteur pour la correspondance

Tél. : +226209722 87; fax : +22620972320

E-mail : bouyer@cirad.fr
}

leurs hôtes. En effet, chez les bovins pâturant sur parcours naturel, ces tiques se fixent provisoirement dans les espaces interdigités avant de gagner, lorsque les animaux se couchent, leurs sites de prédilection (mamelle, périnée...) ; elles peuvent alors être facilement éliminées par un traitement administré par pédiluve $(14,15)$.

De nombreux auteurs ont relevé une dominance marquée des attaques de glossines sur les parties basses des membres, en particulier chez Glossina tachinoides $(3,4,11)$ et $G$. longipennis (9). Au Cirdes, les auteurs ont étudié en étable moustiquaire les zones préférentielles de prise de repas sanguin par G. palpalis gambiensis et $G$. tachinoides, qui provoquent les pertes économiques les plus importantes au Burkina Faso. Les zones potentiellement atteintes lors d'un traitement par pédiluve (membres et, dans une moindre mesure, ventre et poitrail), totalisaient ainsi $84 \pm 8$ p. 100 et $92 \pm 6$ p. 100 des repas pour G. tachinoides et G. palpalis gambiensis (intervalles de confiance avec $\alpha=5$ p. 100) (1). L'impact du traitement de bovins à l'aide d'un pédiluve contenant une solution d' $\alpha$-cyperméthrine (Dominex) a été étudié en étable moustiquaire 
contre ces deux espèces lors d'un précédent essai. Les animaux avaient été traités à la fréquence de passage préconisée pour la lutte contre les tiques en début de saison des pluies (tous les deux jours). Le traitement par pédiluve a provoqué une paralysie des glossines identique à celle observée lors du traitement intégral par pulvérisation manuelle de la même molécule (1). Malgré le rinçage de l'animal traité, un taux de paralysie des glossines supérieur à 90 p. 100 a encore été observé trois jours après le dernier traitement. Par ailleurs, Vale et coll. ont récemment montré l'efficacité d'un traitement ciblé des parties déclives par pulvérisateur manuel contre $G$. pallidipes et $G$. morsitans morsitans $(16,17)$.

Le pédiluve permet le traitement de nombreux animaux en peu de temps (après habituation, 120 animaux passent en moins de 15 min dans l'installation) (15). Il donne aux éleveurs la possibilité de mettre en place de façon pérenne une lutte économiquement viable contre les glossines et les trypanosomoses, d'une part, et contre A. variegatum d'autre part. Ces installations étant de plus en plus utilisées dans le pays pour lutter contre A. variegatum, il est important d'évaluer leur impact contre les glossines.

C'est pourquoi des essais ont été mis en place en conditions expérimentales afin de déterminer l'effet insecticide des traitements réalisés à une fréquence ayant une visée acaricide, de préciser l'intervalle entre traitements à appliquer pour lutter contre les glossines sur le terrain, et pour évaluer l'efficacité et la rémanence d'un traitement pédiluve contenant une suspension aqueuse de deltaméthrine (Vectocid) sur trois espèces de glossines, G. morsitans submorsitans, $G$. tachinoides et $G$. palpalis gambiensis. Ce mode de traitement a été comparé à l'imprégnation totale des animaux par la même formulation, et à la pulvérisation partielle ciblée sur les pattes des bovins.

\section{MATERIEL ET METHODES}

\section{Recueil des données}

Six bovins métis zébu $\times$ baoulé, âgés de 3 à 5 ans et pesant de 250 à $350 \mathrm{~kg}$ ont été utilisés. Un animal a servi de témoin négatif et n'a fait l'objet d'aucun traitement. Un autre animal a été le témoin positif et a été traité intégralement par pulvérisation manuelle de $2 \mathrm{~L}$ de suspension aqueuse de deltaméthrine à la concentration préconisée par le fabricant en Europe ( $1 \mathrm{~mL} / 2$ L, soit 0,0025 p. 100) ainsi qu'à une concentration double $(1 \mathrm{~mL} / \mathrm{L}$, soit 0,005 p. 100), préconisée par le fabricant en Afrique subsaharienne. Deux animaux sont passés trois fois à deux jours d'intervalle dans un pédiluve contenant une suspension aqueuse de deltaméthrine à la même concentration (le niveau de la formulation dans le bac du pédiluve a varié durant l'essai entre 19 et $16 \mathrm{~cm}$ ). Deux animaux ont été traités une fois au niveau des membres jusqu'à l'épaule et la hanche par la même suspension au moyen d'un pulvérisateur manuel (200 mL de suspension). Tous les deux jours, les animaux ont été exposés au soleil pendant $3 \mathrm{~h}$ et arrosés entièrement par $50 \mathrm{~L}$ d'eau pour se rapprocher des conditions naturelles de la saison des pluies et de l'effet de trempage des pieds qui intervient lors de l'abreuvement (effet de rinçage). Des rotations entre les modalités de traitement (tableau I) ont permis de différentier les variations individuelles des effets du traitement. Un délai permettant la disparition de tout effet insecticide a séparé ces rotations.

Afin d'évaluer l'impact des traitements sur les glossines, deux types de contacts entre les mouches et les animaux ont été étudiés. Pour le premier, des cages contenant 25 individus (G. p. gambiensis mâles) ont été régulièrement installées, pendant $10 \mathrm{~min}$, sur huit emplacements précis du corps des bovins (cou, dos, ventre, sections proximales, intermédiaires et distales des pattes avant et arrière) dans le but de comparer l'imprégnation des différentes zones anatomiques par la formulation insecticide ; au total, 252 lots d'insectes ont été utilisés, soit 6302 glossines. Pour le second, 300 glossines (en nombre identique pour les trois espèces) ont été lâchées pendant deux heures (de 8 à $10 \mathrm{~h}$ du matin) en présence d'un des bovins de l'étude dans une étable recouverte d'une moustiquaire. Les insectes ont ensuite été capturés et placés en cages à l'insectarium. Ces lâchers ont été effectués tous les deux jours jusqu'à l'obtention pendant trois séances successives d'un taux de mortalité inférieur à 50 p. 100 pour le traitement le plus rémanent (pulvérisation complète). Deux cent trente-trois lots d'insectes ont été utilisés, totalisant 22082 glossines.

Deux heures après leur capture, toutes les glossines ont été examinées afin d'estimer leur état de gorgement et leur éventuelle paralysie. Ce délai a permis de considérer toutes les glossines encore paralysées comme mortes, ce qui aurait été le cas si elles avaient été soumises aux conditions naturelles (prédation et dessèchement).

En début d'expérience, avant tout traitement, chaque bovin a été présenté aux glossines, dans l'étable moustiquaire, à quatre reprises afin d'évaluer une éventuelle différence d'attractivité entre les animaux. Durant l'expérience, les volières ont été systématiquement mouillées une heure avant chaque lâcher, pour assurer la survie des glossines en augmentant l'humidité ambiante, puis lavées après chaque séance pour éliminer les éventuels résidus d'insecticide. Des lâchers d'une heure (entre 8 et 9 h) ont été effectués en volières vides au cours de l'expérience pour déterminer une éventuelle rémanence des insecticides dans le bâtiment. La température et l'hygrométrie ont été mesurées au cours de toutes les séances de lâchers pour écarter l'effet d'un éventuel « accident » climatique.

\section{Analyse des données}

La variable à expliquer a été le taux de paralysie des glossines après leur exposition aux animaux traités. Ce taux était formé d'un numérateur égal au nombre de glossines paralysées ou mortes après exposition, et d'un dénominateur égal au nombre de glossines contenues dans la cage ou lâchées dans la volière.

Pour l'étude en cages, les variables explicatives ont été :

- la région anatomique où les cages ont été appliquées : «membres » (partie distale et proximale des membres) et « autre régions » (abdomen, corps, cou, tête) ;

- le type de traitement utilisé pour imprégner les bovins selon quatre modalités : une modalité « Témoin » qui ne comprenait pas d'imprégnation, pour montrer l'absence d'insecticide résiduel dans la volière ou sur les bovins utilisés et dont les données n'ont pas été utilisées dans les analyses statistiques; une modalité «Spray partiel » avec application de $200 \mathrm{~mL}$ de suspension insecticide sur

\section{Tableau I}

Plan d'expérience : numéros de boucle des animaux utilisés pour les différentes modalités de traitement

\begin{tabular}{|c|c|c|c|c|}
\hline $\begin{array}{l}\text { Date du } \\
\text { traitement }\end{array}$ & Témoin & $\begin{array}{c}\text { Pulvérisation } \\
\text { partielle }\end{array}$ & Pédiluve & $\begin{array}{l}\text { Pulvérisation } \\
\text { intégrale }\end{array}$ \\
\hline \multicolumn{5}{|c|}{ Traitement simple dose } \\
\hline 29/01/2005 & 196 & 199 & 198 & 197 \\
\hline \multicolumn{5}{|c|}{ Traitement double dose } \\
\hline $25 / 06 / 2005$ & 152 & 199 & 198 & 197 \\
\hline $18 / 10 / 2005$ & 932 & 197 & 196 & 199 \\
\hline
\end{tabular}


les pattes des bovins à l'aide d'un spray manuel ; une modalité "Pédiluve » avec traitement des animaux à l'aide d'un pédiluve ; et une modalité «Spray total » avec aspersion de l'ensemble de l'animal par $2 \mathrm{~L}$ de suspension insecticide à l'aide d'un pulvérisateur manuel ;

- le temps (en jours) depuis l'imprégnation ; les mouches ont été exposées à des bovins imprégnés d'insecticide depuis le lendemain du traitement jusqu'à quatre semaines après cette date.

Pour l'étude en volière, les variables explicatives ont été :

- l'espèce de glossine - G. tachinoides, G. palpalis gambiensis et G. morsitans submorsitans ;

- la dose utilisée pour le traitement, soit 0,0025 p. 100 (modalité

« Simple »), ou 0,005 p. 100 (modalité « Double») ;

- le type de traitement utilisé pour imprégner les bovins, avec les mêmes modalités que dans le cas précédent ;

- le temps depuis l'imprégnation (en jours).

Une analyse exploratoire a été réalisée afin d'étudier la forme et l'intensité de la relation entre les variables explicatives et la variable à expliquer. Un graphe de dispersion a été utilisé pour représenter la relation entre la variable à expliquer et le temps - ajustée par une régression logistique ordinaire, conditionnellement aux autres variables explicatives.

Etant donné la nature de la variable à expliquer (proportion), un modèle de régression logistique a été utilisé pour quantifier l'intensité de sa relation avec les variables explicatives (8). Différents facteurs étaient susceptibles d'introduire une dispersion des données plus grande - phénomène appelé surdispersion - que celle de la loi binomiale souvent utilisée pour modéliser les proportions (10) : répétition des expositions aux mêmes bovins, conditions climatiques variables selon les jours où les essais ont été réalisés, etc. Pour tenir compte de cette éventualité, les auteurs ont utilisé un modèle de régression logistique béta-binomiale (12), sauf dans le cas de l'étude de la variabilité du taux de mortalité liée aux bovins imprégnés où un modèle de régression logistique à effets fixes et aléatoires a été utilisé (5). Avec la paramétrisation retenue pour le modèle béta-binomial, la surdispersion des données (par rapport à une loi binomiale) a été représentée par un coefficient de corrélation intra-groupe $\varphi$.

Différents modèles étaient plausibles et ont été comparés afin de sélectionner le modèle pouvant ajuster correctement les données tout en restant parcimonieux en nombre de paramètres. La qualité de l'ajustement a été vérifiée pour le modèle le plus complexe en calculant la statistique du chi-deux de Pearson (10) et en comparant graphiquement les valeurs observées et prédites. Un modèle plus parcimonieux a ensuite été sélectionné à l'aide du critère d'information d'Akaike (AIC) ou de l'AIC corrigé (AICc) dans le cas de taille d'échantillon $n$ faible par rapport au nombre de paramètres $k$ estimés $(n / k<40)(2)$.

La persistance $\mathrm{T}_{50 \%}$ de l'effet de la deltaméthrine a été estimée à partir de l'équation des modèles : temps au bout duquel on observe encore 50 p. 100 de mortalité chez les glossines exposées à des bovins imprégnés. L'intervalle de confiance a été estimé à l'aide d'une méthode de Monte Carlo.

Afin de comparer l'impact des différents types de traitement sur des populations de mouches en tenant compte des différents rythmes d'administration de la deltaméthrine (chaque quinzaine pour les aspersions complètes et partielles, tous les trois jours pour le pédiluve), la mortalité $M_{1-15}^{t, g}$ d'une cohorte de glossines de l'espèce $g$, nées trois jours avant le premier traitement, a été calculée après $15 \mathrm{j}$ d'exposition à des bovins traités selon la méthode $t$. On a supposé pour cette estimation que les mouches se nourrissaient uniquement sur bovins imprégnés et que l'intervalle entre les repas de sang était constant et d'une durée de quatre jours. Avec cette hypothèse, les glossines de la cohorte se sont nourries aux jours 1 , 5,9 et 13 pour lesquels la mortalité $p_{i}^{t, g}$ était celle prédite par le meilleur modèle de régression béta-binomiale pour la catégorie de traitement $t$, l'espèce de glossine $g$ le $i^{e}$ jour après l'administration du traitement. Pour les autres jours, on a supposé que la mortalité des mouches de la cohorte était nulle. La mortalité $M_{1-15}^{t, g}$ a ainsi été calculée selon la formule :

$$
\left\{\begin{array}{l}
p_{i}^{t, g}=0 \text { si } i \notin\{1,5,9,13\} \\
M_{1-15}^{t, g}=1-\prod_{i=1}^{15}\left(1-p_{i}^{t, g}\right)
\end{array}\right.
$$

Une régression logistique à effets fixes et aléatoires a été effectuée pour étudier la variabilité de la réponse liée à la vache. Pour le lot $i$ de glossines et la vache $j, j \in\{1, \ldots 6\}$, le modèle de la mortalité observée $p_{i, j}$ a été le suivant :

$$
\left\{\begin{array}{l}
p_{i, j}=\pi_{i}+e_{i, j} \\
\operatorname{logit}\left(\pi_{j}\right)=\log \left(\frac{\pi_{j}}{1-\pi_{j}}\right)=X b+Z \hat{u}_{j} \\
u_{j}=\mathrm{N}\left(0, v^{2}\right)
\end{array}\right.
$$

où $\pi_{\mathrm{j}}$ était la mortalité prédite par le modèle, $e_{i, j}$ l'erreur résiduelle, $X$ la matrice du plan d'observation correspondant aux effets fixes du meilleur modèle béta-binomial identifié précédemment, $b$ le vecteur des coefficients de ces effets fixes, $Z$ une matrice colonne unité (ne contient que des 1 ), $u_{j}$ le coefficient de l'effet aléatoire lié à l'intercept du modèle, constant pour la vache $j$ et indépendant de l'effet aléatoire de la vache $k$, avec $j \neq k$, supposé de distribution gaussienne, de moyenne nulle et de variance $v^{2}$ (à estimer).

Les valeurs estimées de la mortalité ont été utilisées pour calculer la mortalité $M^{t, g}(1-15)$ en séparant la variabilité liée aux effets fixes $X \hat{b}$ et à la vache $j Z \hat{u}_{\text {. }}$. Le logiciel $\mathrm{R}$ a été utilisé pour l'analyse des données et les graphiques (13).

\section{RESULTATS}

\section{Expérience en cages}

Le résultat de l'exploration graphique est présenté dans la figure 1. Les glossines mises au contact des parties déclives des animaux ont subi une mortalité plus forte ; l'application de deltaméthrine par pédiluve a entraîné un taux de mortalité plus faible que le spray partiel ou complet, qui a lui-même fait preuve d'une persistance plus forte que l'aspersion partielle. Aucune mortalité n'a été enregistrée avec le témoin négatif : cette catégorie a été éliminée des analyses ultérieures.

Sur la base des résultats de l'exploration graphique, un modèle additif avec des effets principaux « Temps », « Traitement » et « Région anatomique» semblait s'imposer. D'autres modèles ont été comparés à ce modèle additif pour tester l'existence d'une interaction entre le temps et la catégorie de traitement, et entre le temps et la région anatomique. Le modèle comportant tous les effets principaux ainsi que toutes les interactions d'ordre 1 a également été pris en compte pour servir de base aux comparaisons.

Le meilleur modèle au sens de l'AIC a été le modèle comportant tous les effets principaux ainsi que l'interaction entre le temps et le traitement. La qualité globale de l'ajustement du modèle le plus complet n'a pas été mise en doute par le calcul du chi-deux 
$(p=0,67)$. L'estimation du coefficient de corrélation intra-groupe du meilleur modèle béta-binomial a été $\hat{\varphi}=0,64\left(\mathrm{p}<10^{-3}\right)$. Un test du rapport des vraisemblances a permis de confirmer la signification de l'interaction entre le temps et le traitement $(p=0,009)$. La dispersion des réponses est restée forte pour un profil de variables explicatives données, probablement en raison de l'hétérogénéité de l'imprégnation par la deltaméthrine des régions anatomiques classées dans chacune des deux catégories (membres ou autres). L'interaction entre le temps et la région anatomique n'a pas été significative $(\mathrm{p}=0,67)$. L'effet de la région anatomique a été en revanche significatif $\left(p=2.10^{-4}\right)$, de même que l'effet du type de traitement $\left(\mathrm{p}=3 \cdot 10^{-6}\right)$.

Le taux de paralysie des glossines exposées aux bovins traités par pédiluve a été inférieur à celui obtenu lors de la mise en présence des bovins imprégnés par aspersion complète $\left(\mathrm{p}<10^{-3}\right)$. En revanche, la différence de taux de mortalité entre les glossines exposées aux bovins traités par pédiluve et celles en contact avec des animaux ayant subi une aspersion partielle n'a pas été significative (test de Wald : $c=5,6 ; \mathrm{ddl}=2 ; \mathrm{p}=0,062$ ). Comme attendu après l'exploration graphique (figure 1), le taux de mortalité des glossines exposées aux régions anatomiques supérieures a été inférieur à celui observé aux niveau des membres $\left(\mathrm{p}<10^{-3}\right)$. Le tableau II présente une synthèse des résultats sous forme de persistance de l'effet de la deltaméthrine $\left(\mathrm{T}_{50 \%}\right)$.

\section{Expérience en volière}

Le résultat de l'exploration graphique est présenté dans la figure 2. Les glossines exposées aux animaux témoins n'ont pas présenté de mortalité notable, excepté au jour 20 pour l'un d'entre eux (panel supérieur gauche). Cette mortalité a été imputable à une température externe particulièrement élevée ce jour-là. Les glossines exposées aux animaux traités par pulvérisation totale ont connu un taux de mortalité élevé (> 50 p. 100) jusque vers le dixième jour, avec les deux doses de traitement. En revanche, les taux de mortalité sont restés bas pour les animaux imprégnés par aspersion partielle et par pédiluve. Seul le traitement à double dose a permis de maintenir un taux de mortalité supérieur à 50 p. 100 pendant quelques jours. Cette observation a conduit à supposer l'existence

Données observées • Moyenne ajustée

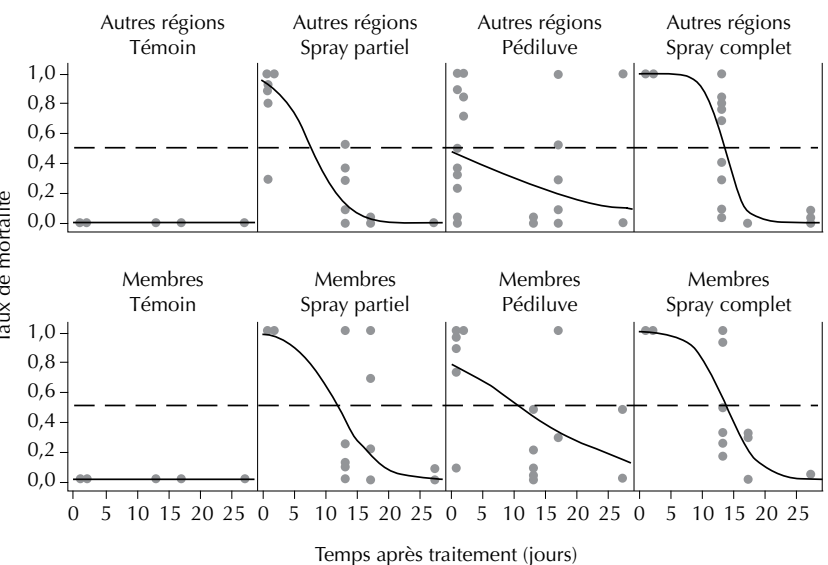

Figure 1 : effet des modalités de traitement des bovins à la deltaméthrine sur la mortalité de Glossina palpalis gambiensis mâles, mis dans des cages contenant 25 mouches, en fonction de la région anatomique d'exposition. L'intersection de la courbe de mortalité moyenne (ajustée avec une régression logistique) avec la ligne horizontale tiretée tracée à $y=0,5$ représente la persistance de l'effet de l'insecticide $\left(T_{50 \%}\right)$. d'une interaction entre les variables temps et dose. La persistance de l'effet de l'insecticide a semblé plus élevée pour les glossines de l'espèce G. morsitans, notamment dans le cas des imprégnations à double dose.

La figure 2 montre l'influence de l'effet du temps et de la catégorie de traitement sur le taux de paralysie des glossines. La variable temps a été décalée d'un jour de manière à avoir son origine au premier jour d'observation (le lendemain de l'imprégnation). La persistance de l'effet de l'exposition a dépendu à la fois du traitement (lecture des panels par ligne), de la dose d'insecticide utilisée pour l'imprégnation (comparaison des courbes dans chaque panel) et de l'espèce de glossine (lecture des panels par colonne). Ces effets supposés ont correspondu à des interactions entre le temps et le type d'imprégnation, entre le temps et la dose d'insecticide, et entre le temps et l'espèce de glossine. L'ajustement des différents modèles possibles et leur comparaison à l'aide de l'AIC indiquait que le meilleur modèle comportait tous les effets fixes ainsi que les interactions Temps*Dose, Temps*Traitement et Temps*Espèce.

\section{Tableau II}

Persistance (en jours) de l'effet du traitement $\left(\mathrm{T}_{50 \%}\right)$ des bovins par la deltaméthrine sur le taux de paralysie des glossines en cages

\begin{tabular}{lccc} 
Type de traitement & $\begin{array}{c}\text { Région } \\
\text { anatomique }\end{array}$ & T $_{\mathbf{5 0} \%}$ & IC $\mathbf{9 5 \%}$ \\
\hline Pulvérisation intégrale & Membres & 18 & $(16,22)$ \\
& Autres & 14 & $(12,16)$ \\
Pulvérisation partielle & Membres & 11 & $(7,17)$ \\
& Autres & 7 & $(4,11)$ \\
Pédiluve & Membres & 4 & $(2,8)$ \\
& Autres & 0 & $(0,2)$
\end{tabular}

IC : intervalle de confiance

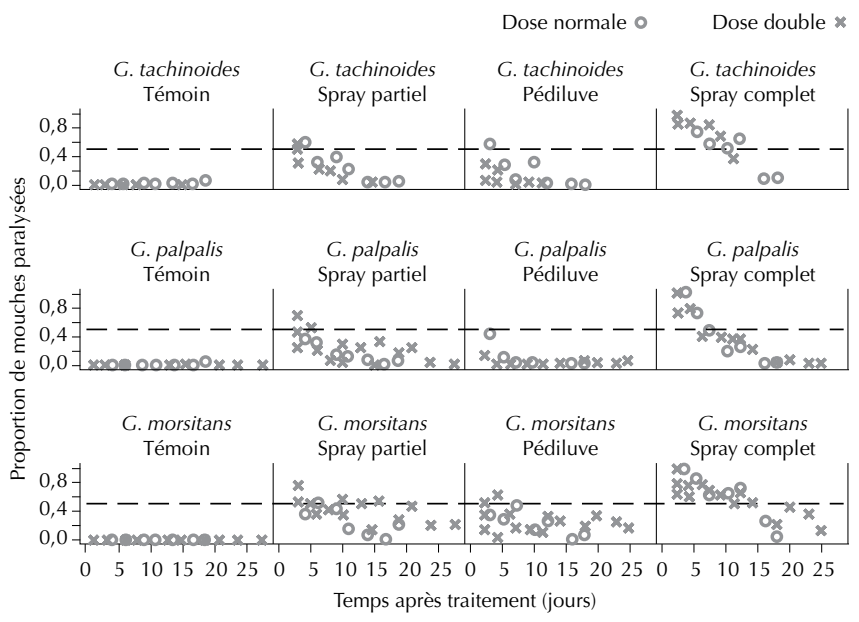

Figure 2 : effet du traitement sur le taux de paralysie des glossines selon leur espèce, la dose de produit actif et les modalités de son application sur les bovins. Les points représentent les données brutes. Les courbes sont les valeurs prédites par une régression logistique de la probabilité de mortalité en fonction du temps dans chaque panel. L'intersection de la courbe de paralysie avec la ligne horizontale tiretée tracée à $y=0,5$ représente la persistance de l'effet de l'insecticide $\left(T_{50 \%}\right)$. 
La qualité globale de l'ajustement n'a pas été mise en doute par le test du chi-deux $(p=0,52)$, et les graphes des valeurs observées et prédites (non montrés ici) ont révélé que l'ajustement des données par le modèle était satisfaisant.

Les coefficients des effets fixes du meilleur modèle béta-binomial sont présentés dans le tableau III. Le coefficient de corrélation intra-groupe estimé a été $\hat{\varphi}=0,09\left(\mathrm{p}<10^{-3}\right)$. Le lendemain de l'imprégnation des bovins à la deltaméthrine, le taux de mortalité des mouches exposées aux bovins traités par pédiluve a été inférieur à celui obtenu par l'exposition aux bovins imprégnés par spray partiel $\left(\mathrm{p}<10^{-3}\right)$. Paradoxalement, le taux de mortalité des glossines exposées à des bovins imprégnés par pulvérisation partielle à la deltaméthrine avec une dose double a été inférieur à celui observé avec une dose normale $\left(\mathrm{p}<10^{-3}\right)$. Le taux de mortalité de G. p. gambiensis a été inférieur à celui de G. m. submorsitans en début d'expérience $\left(\mathrm{p}<10^{-3}\right)$, mais il n'y a pas eu de différence significative entre G. m. submorsitans et G. tachinoides $(\mathrm{p}=0,443)$. L'effet du traitement par pulvérisation totale a diminué plus rapidement que celui obtenu pas spray partiel $\left(\mathrm{p}<10^{-3}\right)$. Le traitement à double dose a permis d'obtenir une diminution plus lente du taux de mortalité qu'avec un traitement à dose normale $\left(\mathrm{p}<10^{-3}\right)$. Enfin, la diminution du taux de mortalité a été plus lente avec G. $m$. submorsitans et plus rapide avec G. tachinoides.

Le tableau IV présente une synthèse des résultats sous forme de persistance de l'effet de la deltaméthrine. L'imprégnation des bovins par pédiluve n'a pas eu d'effet suffisant pour estimer la persistance telle que définie ici. La dose d'insecticide n'a pas eu d'influence dans le cas d'une imprégnation des bovins par spray total. En revanche, la persistance estimée avec le spray total a été environ deux fois plus longue qu'avec le spray partiel.

Les résultats du meilleur modèle béta-binomial utilisé pour calculer la mortalité de cohortes de glossines sont présentés dans le tableau V. A titre de comparaison, les taux de mortalité d'une cohorte de glossines soumise pendant $15 \mathrm{j}$ à des probabilités quotidiennes de mortalité de 1 et 3 p. 100 seraient respectivement de 14,0 et 36,7 p. 100. Le tableau V illustre l'effet compensatoire du passage régulier dans le pédiluve (par rapport aux traitements par aspersion) sur la mortalité cumulée calculée sur $15 \mathrm{j}$. Cette mortalité est restée supérieure à celle nécessaire pour obtenir l'extinction d'une population isolée de glossines $\left(3\right.$ p. $\left.100 \mathrm{j}^{-1}\right)(6,7)$. En revanche, l'utilisation d'une double dose d'insecticide n'a pas semblé être justifiée par les résultats de cette étude.

Afin d'évaluer l'impact des variations liées aux bovins, le même indicateur que celui calculé dans le tableau IV a été estimé à l'aide du modèle de régression logistique à effets fixes et aléatoires. Seuls ont été considérés les traitements effectués avec une dose normale de deltaméthrine. Les résultats sont présentés dans la figure 3. La variabilité liée à la vache a été importante, quelle qu'ait été l'espèce de glossine considérée, et s'est manifestée de manière d'autant plus forte que le taux de mortalité se rapprochait de 50 p. 100.

\section{DISCUSSION}

Les résultats des essais en cage ont présenté une forte variabilité probablement liée à l'inclusion, dans une même catégorie (membres et autres régions), de régions anatomiques subissant une imprégnation insecticide très variable lors des passages dans le pédiluve.

La persistance de la deltaméthrine 0,005 p. 100 (Vectocid) estimée dans cette étude pour les pulvérisations partielle ou totale a été similaire à celle rapportée par Torr et coll. (2007) pour $G$. m. morsitans et G. pallidipes avec du Decatic. Dans le cas

\section{Tableau III}

Coefficients des effets fixes du meilleur modèle bétabinomial du taux de paralysie des glossines après exposition à des bovins imprégnés à la deltaméthrine

\begin{tabular}{lrrr} 
Effet & Estimation & Erreur standard & $\mathbf{P}$ \\
\hline Intercept & 1,06 & 0,28 & $<10^{-3}$ \\
Temps & $-0,18$ & 0,03 & $<10^{-3}$ \\
Pédiluve & $-1,29$ & 0,23 & $<10^{-3}$ \\
Pulvérisation totale & 2,23 & 0,24 & $<10^{-3}$ \\
Double dose & $-1,05$ & 0,24 & $<10^{-3}$ \\
G. palpalis gambiensis & $-0,59$ & 0,24 & 0,014 \\
G. tachinoides & 0,20 & 0,26 & 0,443 \\
Temps * Pédiluve & 0,04 & 0,02 & 0,069 \\
Temps * Spray total & $-0,12$ & 0,02 & $<10^{-3}$ \\
Temps * Double dose & 0,14 & 0,02 & $<10^{-3}$ \\
Temps * G. palpalis gambiensis & $-0,05$ & 0,02 & 0,016 \\
Temps * G. tachinoides & $-0,11$ & 0,03 & $<10^{-3}$
\end{tabular}

\section{Tableau IV}

Persistance de l'effet du traitement $\left(\mathrm{T}_{50 \%}\right)$ des bovins par la deltaméthrine sur le taux de paralysie des glossines

\begin{tabular}{lcccc} 
Espèce & Traitement & Dose & $\mathrm{T}_{\mathbf{5 0} \%}$ (j) & IC 95\% (j) \\
\hline Glossina morsitans & Pulvérisation & Simple & 7 & $(5,9)$ \\
submorsitans & partielle & Double & 1 & $(0,9)$ \\
& Pédiluve & Simple & 0 & $(0,3)$ \\
& & Double & - & - \\
& Pulvérisation & Simple & 12 & $(11,13)$ \\
& totale & Double & 16 & $(14,18)$ \\
G. palpalis & Pulvérisation & Simple & 3 & $(0,5)$ \\
gambiensis & partielle & Double & - & - \\
& Pédiluve & Simple & - & - \\
& & Double & - & - \\
& Pulvérisation & Simple & 9 & $(8,10)$ \\
& totale & Double & 9 & $(8,10)$ \\
& & & & \\
G. tachinoides & Pulvérisation & Simple & 5 & $(4,7)$ \\
& partielle & Double & 2 & $(0,5)$ \\
& Pédiluve & Simple & 1 & $(0,3)$ \\
& & Double & - & - \\
& Pulvérisation & Simple & 10 & $(9,11)$ \\
& totale & Double & 10 & $(9,12)$
\end{tabular}

IC : intervalle de confiance

du traitement par pédiluve, elle a été en revanche inférieure à celle observée dans les mêmes conditions au Cirdes pour l' $\alpha$-cyperméthrine 0,005 p. 100 (Dominex), alors que les résultats étaient similaires pour le traitement intégral (1). Il est possible que la deltaméthrine, sous cette forme, soit moins stable que l' $\alpha$-cyperméthrine lorsqu'elle séjourne dans de l'eau souillée (boue et bouse s'accumulent rapidement dans le pédiluve). Il serait utile 
de confirmer cette hypothèse par une étude expérimentale et, le cas échéant, de rechercher une formulation permettant d'améliorer cette stabilité.

Malgré une efficacité inférieure à celle obtenue avec une administration par spray, il semble possible d'envisager le contrôle de populations sauvages de glossines de ces trois espèces à l'aide de suspension de deltaméthrine administrée à des bovins par pédiluve, en particulier lorsque celui-ci est utilisé tous les trois jours, comme c'est le cas dans le cadre de la lutte acaricide. Un suivi de terrain d'une population de glossines dans le village de Dafinso avait d'ailleurs révélé une réduction rapide et significative des densités de glossines sauvages (G. p. gambiensis et G. tachinoides) (1). La

\section{Tableau V}

Taux de mortalité de cohortes de glossines exposées à des bovins imprégnés à la deltaméthrine pendant

$15 \mathrm{j}$ en fonction du type de traitement, sous I'hypothèse d'un cycle trophogonique de $4 \mathrm{j}$, d'une mortalité quotidienne prédite par le meilleur modèle

béta-binomial pour les jours de repas sanguins et nulle les autres jours

$\begin{array}{lccc}\text { Espèce } & \text { Traitement } & \text { Dose } & \text { Mortalité (\%) } \\ \text { Glossina morsitans } & \text { Pulvérisation } & \text { Simple } & 93,5 \\ \text { submorsitans } & \text { partielle } & \text { Double } & 89,9 \\ & \text { Pédiluve } & \text { Simple } & 85,9 \\ & & \text { Double } & 62,6 \\ & \text { Pulvérisation } & \text { Simple } & 99,9 \\ \text { G. palpalis } & \text { totale } & \text { Double } & 99,7 \\ \text { gambiensis } & \text { Pulvérisation } & \text { Simple } & 77,7 \\ & \text { partielle } & \text { Double } & 66,6 \\ & \text { Pédiluve } & \text { Simple } & 67,3 \\ & & \text { Double } & 41,1 \\ & \text { Pulvérisation } & \text { Simple } & 98,8 \\ & \text { totale } & \text { Double } & 97,3 \\ & & & \\ \text { G. tachinoides } & \text { Pulvérisation } & \text { Simple } & 89,4 \\ & \text { partielle } & \text { Double } & 80,7 \\ & \text { Pédiluve } & \text { Simple } & 86,3 \\ & & \text { Double } & 63,1 \\ & \text { Pulvérisation } & \text { Simple } & 99,6 \\ & \text { totale } & \text { Double } & 99,1\end{array}$

IC : intervalle de confiance

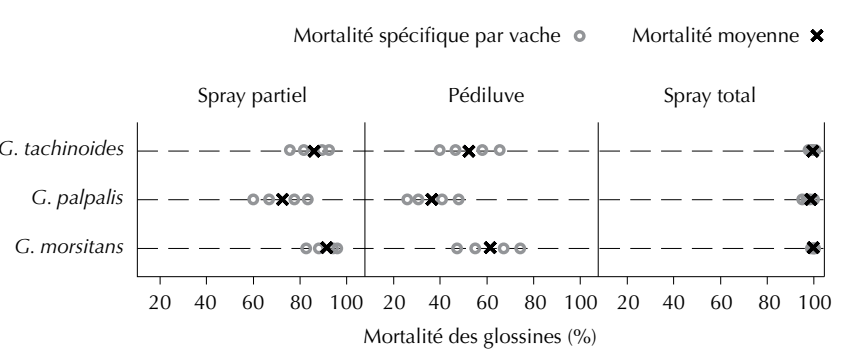

Figure 3 : taux moyen de mortalité cumulée sur 15 jours, et variations liées aux animaux, d'une population de glossines se nourrissant sur des bovins traités par différentes modalités d'application de la deltaméthrine. pulvérisation partielle est encore plus efficace, probablement liée à une meilleure efficacité de la deltaméthrine non souillée, et pourrait donc être utilisée par les éleveurs nomades. Cependant, elle nécessite un temps de traitement plus important et une contention efficace des animaux, sans quoi une grande proportion du produit est perdue.

Au-delà de la réduction du coût en insecticides lié à l'utilisation du pédiluve, le gain de temps procuré par cette méthode est intéressant pour les agroéleveurs, notamment en saison des pluies, moment où ils sont très occupés par les cultures (15). Son efficacité contre $A$. variegatum (15) en fait par ailleurs une technique de lutte intégrée contre les vecteurs, qui a déjà été choisie par des projets de développement au Burkina Faso. Par ailleurs, des travaux sont en cours pour évaluer l'effet de ces traitements par pédiluve sur la transmission de la trypanosomose bovine.

\section{CONCLUSION}

La mortalité imposée aux glossines par le pédiluve permet de supposer que cette technique est utilisable pour la lutte préventive contre les trypanosomoses bovines transmises par les glossines dans certaines conditions. Il est cependant nécessaire d'étudier l'impact de cette technique sur l'incidence des trypanosomoses en milieu réel et dans différentes conditions environnementales pour en évaluer l'impact définitif. De plus, il est nécessaire de développer «l'emballage » de la technique qui reste difficile à utiliser pour les populations illettrées, en collaboration avec des chercheurs des sciences sociales. Il est déjà prévu de construire des pédiluves et de tester leur adoption dans le cadre de divers projets de développement au Burkina, Tchad, Mali, Bénin et Sénégal.

\section{Remerciements}

Nous remercions la société Ceva Santé animale (Libourne, France) pour le financement de cet étude. Nous remercions également le Cirdes qui a mis à notre disposition ses installations et a fourni les glossines utilisées pour les essais, et plus particulièrement S. Boli, B. Cene, S.P. Kaboré, A. Sana, L. Sanogo, F. Sanou, W. Yoni et S. Zoungrana.

\section{BIBLIOGRAPHIE}

1. BOUYER J., STACHURSKI F., KABORE I., BAUER B., LANCELOT R., 2007. Tsetse control in cattle from pyrethroid footbaths. Prev. vet. Med., 78: 223-238.

2. BURNHAM K.P., ANDERSON D.R., 2002. Model selection and multimodel inference: a practical information-theoretic approach, $2^{\text {nd }}$ Edn. New York, NY, USA, Springer-Verlag.

3. CHALLIER A., 1973. Ecologie de Glossina palpalis gambiensis Vanderplank, 1949 (Diptera-Muscidae) en savane d'Afrique occidentale. Paris, France, Orstom, 274 p. (Mémoires, vol. 64)

4. DAVIES H., 1967. Les glossines dans le Nord-Nigeria. Manuel destiné au personnel de lutte contre les glossines. Maisons-Alfort, France, lemvt, $260 \mathrm{p}$.

5. DIGGLE P.J., HEAGERTY P., LIANG K., ZEGER S., 2002. Analysis of longitudinal data, $2^{\text {nd }}$ Edn. Oxford, UK, Oxford University Press.

6. HARGROVE J.W., 1988. Tsetse: the limits to population growth. Med. vet. Entomol., 2: 203-217.

7. HARGROVE J.W., 2003. Tsetse eradication: sufficiency, necessity and desirability. Edinburgh, UK, CTVM

8. HOSMER D.W., LEMESHOW S., 2000. Applied logistic regression, $2^{\text {nd }}$ Edn. New York, NY, USA, John Wiley \& Sons. 
9. MAKUMI J.N., GREEN C.H., BAYLIS M., 1996. The role of cattle as hosts of Glossina longipennis at Galana Ranch, South-Eastern Kenya. Med. vet. Entomol., 10: 331-336.

10. MCCULLAGH P., NELDER J.A., 1989. Generalized linear models, $2^{\text {nd }}$ Edn. London, UK, Chapman \& Hall.

11. NASH M.L., HUNGERFORD L.L., NASH T.G., ZINN G.M., 1996. Risk factors for perinatal and postnatal mortality in lambs. Vet. Rec., 139: $64-67$.

12. PRENTICE R.L., GLOECKLER L.A., 1978. Regression analysis of grouped survival data with application to breast cancer data. Biometrics, 34: 57-67.

13. R DEVELOPMENT CORE TEAM, 2007. R: A language and environment for statistical computing. Vienna, Austria, R Foundation for Statistical Computing.
14. STACHURSKI F., 2000. Invasion of West African cattle by the tick Amblyomma variegatum. Med. vet. Entomol., 14: 391-399.

15. STACHURSKI F., LANCELOT R., 2006. Footbath acaricide treatment to control cattle infestation by the tick Amblyomma variegatum. Med. vet. Entomol., 20: 402-412.

16. TORR S.J., MAUDLIN I., VALE G.A., 2007. Less is more: restricted application of insecticide to cattle to improve the cost and efficacy of tsetse control. Med. vet. Entomol., 21: 53-64.

17. VALE G., 2003. Pyrethroid-treated cattle for tsetse control: effect of restricted application. Newsl. Integrated Control Pathog. Trypanosomes Vectors, 7: 15-16.

Reçu le 11.03.2008, accepté le 11.06.2009

\section{Summary}

Bouyer J., Stachurski F., Gouro A., Lancelot R. On-Station Cattle Insecticide Treatment against Tsetse Flies using a Footbath

The study of arthropod vector behavior can lead to inexpensive and innovative control techniques for farmers. For instance, treating cattle feet with an insecticide footbath eliminates Amblyomma variegatum (Acari: Ixodidae) ticks captured in the pasture, before they can reach their predilection sites. The authors recently showed that treating cattle with a formulation of $\alpha$-cypermethrin in a footbath was as efficient as full spraying to control Glossina tachinoides Westwood, 1850 (Diptera, Glossinidae) and G. palpalis gambiensis Vanderplank 1949. Here, the authors compared the knock-down efficacy of a deltamethrin formulation (Vectocid) applied as full or partial spray, or in a footbath, against these two species and G. morsitans submorsitans Newstead 1910, in experimental conditions (exposure to tsetse flies in cage, and in the stable under mosquito netting). When exposing cattle to flies in cages, the knock-down effect was similar $(p=0.062)$ for the footbath and the partial spraying, but lower than with full spraying $\left(p<10^{-3}\right)$. In-stable, the footbath treatment was less efficacious than that of the other two techniques $\left(p<10^{-3}\right)$. Nonetheless, fly mortality obtained by footbath was higher than that needed to eliminate an isolated tsetse population $\left(>3 \%\right.$ day $\left.^{-1}\right)$, when assessed over 15 days using a three-day treatment frequency. The footbath treatment may thus prevent tsetse-transmitted cattle trypanosomose infection under some conditions.

Keywords: Cattle - Glossina - Synthetic pyrethrin - Disease control - Spraying - Trypanosomosis - Experimental design.

\section{Resumen}

Bouyer J., Stachurski F., Gouro A., Lancelot R. Tratamiento insecticida de bovinos contra las glosinas mediante pediluvio bajo condiciones experimentales

El estudio del comportamiento de los artrópodos vectores puede llevar al establecimiento de métodos de lucha innovadores y económicos para los criadores. De esta manera, el tratamiento orientado a las extremidades de los miembros de los bovinos mediante un pediluvio acaricida permite eliminar las garrapatas Amblyomma variegatum (Acari: Ixodidae) capturadas en el potrero, antes de que accedan a sus sitios de fijación definitivos. Los autores mostraron recientemente que con una solución de $\alpha$-cypermetrina, el tratamiento por pediluvio fue tan eficaz como una pulverización integral para luchar contra Glossina tachinoides Westwood, 1850 (Diptera, Glossinidae) y G. palpalis gambiensis Vanderplank 1949. Los autores comparan aquí bajo condiciones experimentales (jaulas y establo con mosquitero) la eficacia de una formulación de deltametrina (Vectocid) administrada mediante pulverización integral, parcial o por pediluvio, eficacia que se tradujo por la parálisis de estas dos especies y de G. morsitans submorsitans Newstead 1910 cuando se pusieron en presencia de los animales tratados. Durante la exposición a las glosinas en jaula, las tasas de parálisis de las glosinas expuestas a los bovinos impregnados por pediluvio fue similar a las obtenidas por pulverización parcial ( $p=0,062$ ), pero inferior al obtenido por aspersión completa $\left(p<10^{-3}\right)$. En pajareras, fue inferior al de las dos otras modalidades de tratamiento $\left(p<10^{-3}\right)$. A pesar de esto, la mortalidad debida al pediluvio, calculada sobre 15 días con una frecuencia de tratamiento de tres días, se mantuvo superior a la necesaria para obtener la extinción de una población aislada de glosinas $\left(3 \% \mathrm{~d}^{-1}\right)$. Esta técnica podría ser eficaz en la lucha preventiva contra las tripanosomosis bovinas transmitidas por las glosinas bajo ciertas condiciones.

Palabras clave: Ganado bovino - Glossina - Piretrina sintética Control de enfermedades - Pulverización - Tripanosomosis Diseño experimental. 